\title{
ADAPTIVE PROTECTION SCHEME FOR MVC-ENCODED STEREOSCOPIC VIDEO STREAMING IN IP-BASED NETWORKS
}

\author{
César Díaz, Julián Cabrera, Fernando Jaureguizar, and Narciso García
}

\begin{abstract}
We present an adaptive unequal error protection (UEP) strategy built on the 1-D interleaved parity Application Layer Forward Error Correction (AL-FEC) code for protecting the transmission of stereoscopic 3D video content encoded with Multiview Video Coding (MVC) through IP-based networks. Our scheme targets the minimization of quality degradation produced by packet losses during video transmission in time-sensitive application scenarios. To that end, based on a novel packet-level distortion model, it selects in real time the most suitable packets within each Group of Pictures (GOP) to be protected and the most convenient FEC technique parameters, i.e., the size of the FEC generator matrix. In order to make these decisions, it considers the relevance of the packet, the behavior of the channel, and the available bitrate for protection purposes. Simulation results validate both the distortion model introduced to estimate the importance of packets and the optimization of the FEC technique parameter values.
\end{abstract}

Index Terms - Multiview Video Coding (MVC), video streaming, distortion, Unequal Error Protection (UEP), Application Layer Forward Error Correction (AL-FEC)

\section{INTRODUCTION}

Stereoscopic video transmission is increasingly moving from a frame compatible format, where $3 \mathrm{D}$ content is downsampled to suit existing 2D coding and transmission systems, to a service compatible format, which aims at maintaining the full resolution of the views to provide the user with a high quality $3 \mathrm{D}$ representation. In that sense, MVC is capable of exploiting not only temporal and spatial but also inter-view redundancy, enabling efficient coding of multiple temporally synchronized video sequences, and generating, as a result, a single bitstream [1].

In this paper, we focus on low-latency real-time MVC-encoded stereoscopic 3D video transmission over IP-based networks. This environment is necessarily conditioned by the adverse effects introduced by the communication channel: bursty packet loss and latency, which lead to Quality of Experience (QoE) degradation. Due to the time-sensitive nature of the scenario, Real-time Transmission Protocol (RTP) over UDP is used for transmission. Therefore, external error control mechanisms need to be introduced [2].

It is well known that different parts of the encoded video stream are of unequal importance to the overall 3D perception due to error propagation. Moreover, channel bitrate budget limitations might constrain the resources available for protection purposes. Hence, unequal error protection (UEP) schemes are usually introduced to face the unreliability of the channel [3]. These smart schemes present strategies to decide which part of the data should be protected and how, so that resource availability is not exceeded and the overall quality after decoding is kept as high as possible.

Different UEP strategies designed to protect the transmission of MVC-encoded stereoscopic content have been proposed in the literature [4], [5], [6], [7]. As for monoscopic transmission, they usually differ in two key aspects: the basic data unit considered, and the arrangement of the protection technique [3]. The first one refers to the structure of data whose features are employed for analysis and decision taking within the strategy. A basic unit may consist of: a macroblock, a slice, a transmission packet, a frame, a tempo$\mathrm{ral} / \mathrm{spatial} /$ quality layer, a view... In general, the finer the granularity of evaluation is, the more computationally costly the technique is. In contrast, the coarser the granularity is, the less precise the scheme becomes. The second aspect alludes to how the protection resources are assigned to data, and the way through which protection is generated.

In order to take decisions, these strategies pose distortion minimization problems in which the data unit features, usually along with some other information involving the video transmission system, are included. The solutions to this problems determine the protection policies to follow.

We present a low-complexity adaptive UEP scheme for MVCencoded stereoscopic 3D video streaming over packet networks, the Stereoscopic Video Adaptive Protection (SVAP) scheme. This strategy aims at maximizing the decoded video quality in a real-time scenario. Therefore, its basic unit of analysis and decision is the RTP-wrapped packet, as it presents a good trade-off between key data accessibility (and therefore computational cost lightening), and accuracy of the information obtained in regard to quality degradation. Working at a packet level does not require to our algorithm any further process than that of parsing RTP and Network Abstraction Layer Unit (NALU) headers, which makes it suitable to fulfill transmission delay constraints.

Our algorithm selects in real time the most suitable packets to get protection and the most convenient FEC technique parameters to carry out the generation of the protection stream. To this purpose, it takes decisions considering: (i) the characteristics of the encoded video stream; (ii) the behavior of the transmission channel; and (iii) the bitrate limitations. In order to infer the relevancy of each packet in terms of capacity of causing error propagation, a novel packetlevel distortion model is proposed.

The rest of the paper is organized as follows. In section 2 , the main actors of the video streaming system are described. Section 3 presents the proposed packet-level distortion model. Next, in section 4 , the proposed SVAP scheme is introduced. Simulations and results are presented in section 5 . Finally, in section 6 , we include the conclusions of the paper. 


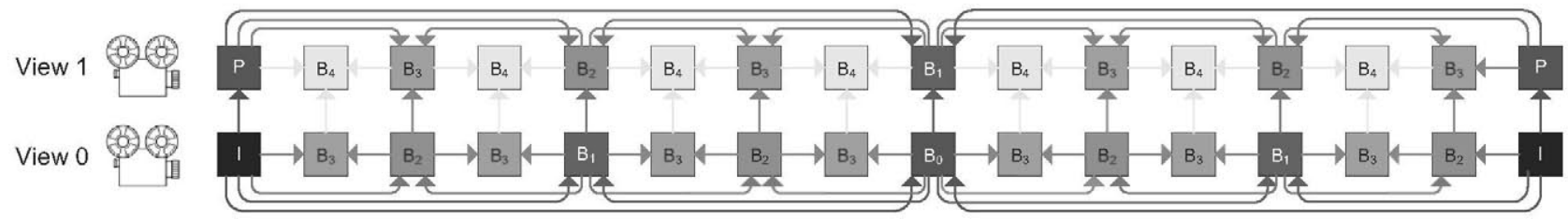

Fig. 1. GOP coding prediction structure

\section{VIDEO STREAMING SYSTEM OVERVIEW}

The main actors in any video streaming system are the encoded video stream and the transmission channel. Therefore, the protection strategy is formulated through the description of their behavior. This characterization is presented next.

\subsection{MVC-encoded Stereoscopic Video Stream}

The sequences corresponding to the two different views in a stereoscopic video are encoded with H.264/MVC employing a specific GOP coding prediction structure, resulting in a single bitstream. A coding prediction structure, as the one presented in Fig. 1, establishes the frame coding dependencies within a GOP. These dependencies utterly determine the relative importance of the different frames in terms of error propagation [8].

The obtained bitstream is finally packetized into RTP packets. Thus, the information associated with a certain frame is carried by a number of data packets. Hence, the video stream packets are of unequal relevance regarding the importance of the information they transport in relation to the coding structure.

Some of the fields included in the RTP and NALU headers give information on the type of frame transported and on the position that the packet holds along the sequence. This information, which can be easily accessed by just parsing the mentioned packet headers, is used by our algorithm to carry out the protection scheme.

\subsection{Communication Channel}

At a packet level, IP-based networks behave as erasure channels, i.e., packets are either lost or received unaltered. Moreover, packet losses in these networks follow a bursty basis [9]. These bursts can be described in terms of frequency of occurrence and length, leading to the fully characterization of the channel's behavior by means of the Packet Loss Rate (PLR) and the Average Burst Loss (ABL).

This description enables the generation of models from which it is possible to predict to some extent the behavior of the channel at a packet level. One of the most widely used models is the simplified Gilbert model [10]. It consists of two states: state G, which indicates successful transmission of the packet, and state $\mathrm{B}$, which indicates packet loss.

\section{PACKET-LEVEL DISTORTION MODEL}

In this section, the proposed packet-level distortion model, jointly with the analysis carried out to its determination, is described.

The distortion model has been inferred from weighting the effects of packet losses and the subsequent error propagation in H.264/MVC-encoded stereoscopic video sequences. To do so, we have first selected several sets of GOPs encoded using different prediction structures. Next, for each GOP, we have carried out a number of tests equal to the number of packets within, each one consisting on dropping the corresponding packet and decoding the resulting sequence. Then, the average mean squared error (MSE) value between the original and every obtained sequence has been calculated. So, these values express how distorting the loss of the different packets within the GOP are. For simplicity, just one slice has been used per frame. Additionally, the error concealment algorithm applied during decoding replaces the lost macroblocks with the ones located in the same regions of its immediately previously decoded reference frame. In the case of I-frames, the algorithm uses the temporally preceding closest frame in the same view that does not use the I-frame as reference.

Figure 2(a) presents the average MSE (vertical axis) obtained after dropping each of the packets (horizontal axis) in a GOP with a prediction structure as the one shown in Fig. 1. The pictures to which each packet belong are indicated for better understanding. All the sequences analyzed present an analogous behavior to the one observed within the presented GOP in terms of distortion.

Three types of error propagation take place within a GOP: intraframe, inter-frame and inter-view. The description and analysis of the three is included next:

-Intra-frame error propagation: looking at Fig. 2(a) one can realize that, within any of the frames, the distortion introduced by the loss of a packet decreases as that packet is located closer to the end of the frame. That can be explained through the fact that when losing a portion of a bitstream, the decoder discards all the succeeding bits until the following synchronization marker arrives. This happens by the arrival of the next NALU header, i.e., by the beginning of the subsequent frame, given that just one slice is used per frame. So, the closer the loss is to the end of the frame, the less data is discarded, and, hence, the lower the distortion introduced.

-Inter-frame error propagation: the quality degradation resulted from the loss of a packet of a picture increases with the amount of information whose decoding process depends on it. Indeed, in Fig. 2(a) it can be observed that the larger the number of frames using a given one as reference is, the greater the distortion caused by the loss of data of the reference picture in the decoded video becomes.

-Inter-view error propagation: same description as for interframe error propagation. Given that the prediction relationship between two frames in different views is of the same nature as two frames in the same view, inter-view error propagation will be considered within inter-frame error propagation.

Based on the analysis presented above, we propose a distortion model of the stereoscopic video packet stream that considers both types of error propagation.

Regarding inter-frame error propagation, it is presumed that the information transported by packets that belong to the same frame approximately affects the same number of macroblocks of the picture. Hence, they can be considered equally important. Therefore, it is assumed that the inter-frame error propagation provoked by the loss of a certain packet does not depend on the position of the packet along the frame, but only on the number of frames that depend on this one 


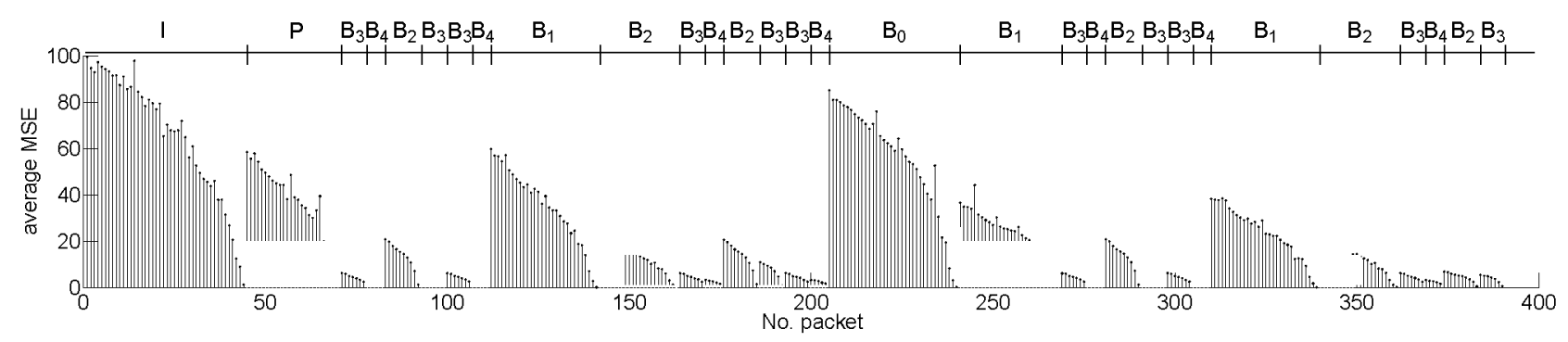

(a) Study of the effect of packet dropping on video quality

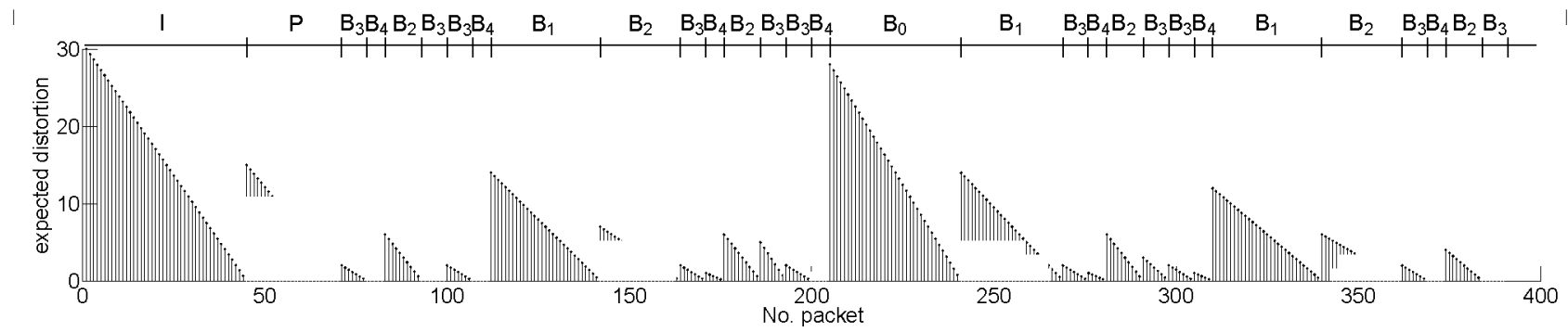

(b) Proposed distortion model

Fig. 2. Distortion introduced by the loss of each packet in the GOP. Darker grey indicates a packet transporting information of the main view, and lighter grey of the inter-view. The type of picture is identified regarding the coding structure presented in Fig. 1

for decoding, disregarding the view they belong to.

As for intra-frame error propagation, the expected distortion introduced by a lost packet is assumed to linearly decrease with the position of the packet along the frame.

So, according to the aforementioned characterization, the proposed distortion model can be fully described by means of three different packet features. In the $k^{\text {th }}$ GOP, the features of the $N_{\text {packets }}$ packets are arranged in three $N_{\text {packets }}$-component vectors: (i) $\boldsymbol{l}_{k}$, where $l_{k}(i)$ represents the position of the $i^{\text {th }}$ packet in the GOP along the frame it belongs to; (ii) $\boldsymbol{s}_{k}$, where $\boldsymbol{s}_{k}(i)$ is the size of this frame (defined as the number of packets belonging to it); and (iii) $\boldsymbol{n}_{k}$, where $\boldsymbol{n}_{k}(i)$ the number of frames depending of this one for decoding (including itself).

Assumed known the GOP coding structure, all the necessary information is collected by just fetching the demanded data from the packet headers. The accessed data helps identify the dependencies of every frame, and, by simple counting, estimate their size.

The expected distortion introduced by the different packets in the $k^{\text {th }}$ GOP due to inter-frame error propagation is given by the

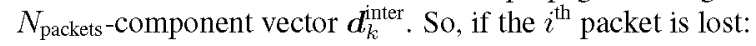

$$
d_{k}^{\text {inter }}(i)=n_{k}(i)
$$

Likewise, the expected distortion introduced by the packets in the $k^{\text {th }}$ GOP due to intra-frame error propagation is presented by the

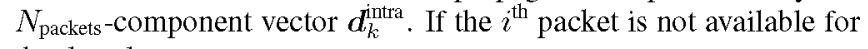
the decoder:

$$
\boldsymbol{d}_{k}^{\text {intra }}(i)=1-\left(\frac{\boldsymbol{l}_{k}(i)}{\boldsymbol{s}_{k}(i)}\right)
$$

As indicated, due to intra-frame error propagation, if a packet is lost, the information carried by the following packets in the frame turns useless for all practical purposes. This induced 'lost' information also becomes a source of inter-frame error propagation. Hence, the total expected distortion introduced by the packets in the $k^{\text {th }}$ GOP

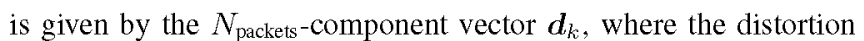
due to not receiving the $i^{\text {th }}$ packet is:

$$
\boldsymbol{d}_{k}(i)=\boldsymbol{d}_{k}^{\text {inter }}(i) \boldsymbol{d}_{k}^{\text {intra }}(i)=\boldsymbol{n}_{k}(i)\left(1-\left(\frac{\boldsymbol{l}_{k}(i)}{\boldsymbol{s}_{k}(i)}\right)\right)
$$

Results of the application of the proposed distortion model are included in Fig. 2(b) for comparison with the real values obtained during the study.

Although MSE objective measures were used for the creation of the distortion model, we do not miss the fact that packet losses in stereoscopic video streaming might especially dramatically affect the perceived quality, even if low MSE values are obtained, as they may lead to binocular rivalry and important visual discomfort [11]. Hence, there exist 3D-adapted error concealment methods that can be applied to mitigate these negative effects [12]. Those strategies are out of the scope of this paper.

\section{PROPOSED SVAP SCHEME}

\subsection{General Description}

The main goal of the proposed protection scheme is to select the most suitable protection policy to minimize the deterioration of the transmitted stereoscopic video caused by packet losses. The chased protection policy designates the most convenient packets to get protection and the optimal FEC parameters to that end. Due to the timesensitive nature of the scenario, this goal is independently pursued within all GOPs in the sequence, as this operating window presents a good trade-off between constituting a representative sample, rapid adaptability, and fulfilling real-time constraints.

In order to find the most appropriate protection policy, a cost minimization problem is posed. Within this problem, first, the intrinsic distortion value of all packets is estimated through the proposed distortion model. These values point out the relative relevance of every packet within the GOP from the point of view of the QoE degradation that its loss might cause. Next, an iterative search of the most 
appropriate FEC parameters is carried out. The result of this pursue is conditioned by the relative relevance of the packets, the recovery capability of the FEC code, the behavior of the transmission channel, and the imposed protection bitrate limitation. The parameters with which the minimum overall distortion is introduced are selected and the most important video data packets are picked to be protected.

\subsection{AL-FEC technique}

The protection packets are generated by means of the AL-FEC scheme proposed by the Pro-MPEG Forum in its COP \#3 r2 [9]. This code is based on the arrangement of data packets in matrices of NR rows and NC columns and the possibility of generating FEC packet both column- and row-wise. The reason for this choice is the simplicity and speed involved in XOR operation coding, and its suitability to deal with bursty loss channels when the appropriate NR and NC are selected.

We have opted to generate protection only column-wise, as illustrated in Fig. 3, due to the fact that these FEC packets offer comparatively greater recovery capacity than row-wise created FEC packets, as the former can cope with loss bursts thanks to interleaving.

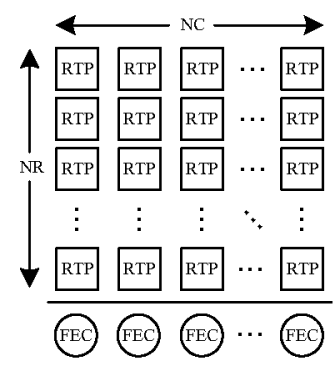

Fig. 3. 1-D interleaved XOR matrix

In the literature there are studies testing the performance of this FEC generation scheme and comparing it to other techniques in terms of the packet recovery rate reached when applying a certain redundancy [13], [14]. These studies show its high efficiency at low PLRs, becoming less efficient as this parameter's value increases. However, in the context of multimedia streaming, the efficiency of a technique is better measured in terms of the video quality achieved given a fixed code rate, since this is its ultimate goal. In that sense, given the unequal importance of video packets in terms of expected distortion, and assuming a fixed code rate, a smart distribution of the redundancy among data can lead to a significant efficiency increase with respect to non-smart schemes, even at the expense of a lower packet recovery rate.

So, with the objective of improving the efficiency of standard 1-D interleaved XOR from the point of view of minimizing the degradation caused by packet losses on the decoded video, our algorithm aims at devoting all the available redundancy to protect the subset of the most distorting packets in the GOP, whereas not generating protection for the less important. For each GOP, just one matrix is used. NC is first fixed to the maximum possible value according to the imposed code rate to allow maximum channel error decorrelation. Hence, devoting more redundancy in practice means employing matrices of a lower NR, which increases the probability of packet recovery, as losing more than one packet per column (which prevents all of them from being recovered) becomes less likely. The optimal matrix size for each GOP minimizes the expected overall distortion within this frame structure.
In addition, since the most convenient packets to be protected within a GOP may not lay sequentially in the bitstream, we allow the layout of non-consecutive packets in the matrix.

\subsection{SVAP Algorithm Description}

Algorithm 1 sketches the operation of the SVAP algorithm, in which the cost minimization problem posed by the protection strategy is solved.

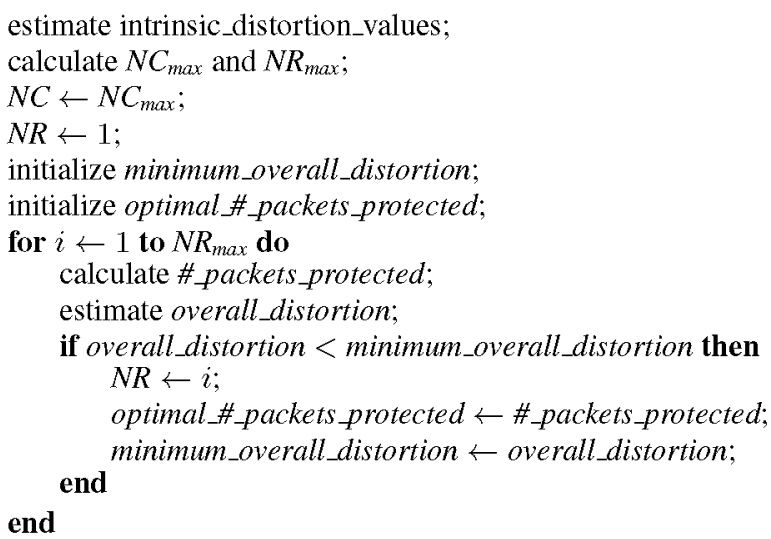

Algorithm 1: Algorithm carrying out the proposed protection scheme

The matrix size with which the minimum overall expected distortion is introduced is chosen for the protection of video packets in the analyzed GOP. The NC. NR most suitable packets, i.e. those that introduce the greatest expected distortion considering the proposed distortion model, are selected to be protected.

The analytical description of the process, including how the overall expected distortion is estimated, is included in the following section.

\subsection{Cost Minimization Problem}

At the $k^{\text {th }}$ GOP, the cost to be minimized, $C_{k}$, is equal to the sum of the expected distortion introduced by each of the $N_{\text {packets }}$ packets belonging to that GOP, $\boldsymbol{D}_{k}(i)$, where $1 \leq i \leq N_{\text {packets }}$ :

$$
\begin{aligned}
& C_{k}=\sum_{i=1}^{N_{\text {packets }}} \boldsymbol{D}_{k}(i) \\
& \text { s.t. }\left(\frac{N_{\text {packets }}}{N_{\text {packets }}+\mathrm{NC}}\right) \geq r_{\mathrm{FECmax}}
\end{aligned}
$$

The condition to which the cost function is subjected expresses the imposition of a maximum bitrate for protection purposes. $r_{\mathrm{FECmax}}$ represents the maximum FEC code rate allowed to fulfill this constraint.

The expected distortion introduced by a packet represents the potential quality degradation of the decoded video stream due to the transmission process of this packet. That is expressed for the $i^{\text {th }}$ packet as:

$$
\boldsymbol{D}_{k}(i)=\boldsymbol{D}_{k}^{\mathrm{G}}(i) \boldsymbol{P}_{k}^{\mathrm{G}}(i)+\boldsymbol{D}_{k}^{\mathrm{B}}(i) \boldsymbol{P}_{k}^{\mathrm{B}}(i)
$$

where $\boldsymbol{D}_{k}^{\mathrm{G}}(i)$ represents the distortion introduced if the packet is satisfactorily transmitted, $D_{k}^{\mathrm{B}}(i)$ indicates the distortion introduced if 
the packet is lost, and $\boldsymbol{P}_{k}^{\mathrm{G}}(i)$ and $\boldsymbol{P}_{k}^{\mathrm{G}}(i)$ are, respectively, the likelihood of these two complementary events.

Since we are facing an erasure channel, the received packets reach the decoder unchanged. Therefore, $D_{k}^{\mathrm{G}}(i)=0$.

Thus, the expected distortion of a packet only considers the expected degradation due to its possible loss in the transmission. The distortion introduced if the packet is lost only depends on its intrinsic distortion value, $\boldsymbol{d}_{k}(i)$, which is estimated employing the proposed distortion model, described in 3 :

$$
\boldsymbol{D}_{k}^{\mathrm{B}}(i)=\boldsymbol{d}_{k}(i)
$$

On the other hand, the likelihood of losing a packet considers the likelihood of not receiving it, PLR, and that of not being able to recover it, $\boldsymbol{P}_{\text {loss. }}$. Hence, it relies on whether this packet is decided to be protected, and on the recovery capability offered by the FEC technique, given the behavior of the channel. The decisions taken over the packets are arranged in the $N_{\text {packets }}$-component vector $\pi_{k}$, so that $\pi_{k}(i)$ is equal to 1 if the $i^{\text {th }}$ packet is selected to be protected, and equal to 0 otherwise. So:

$$
\boldsymbol{P}_{k}^{\mathrm{B}}(i)=\mathrm{PLR} \cdot \boldsymbol{P}_{\text {loss }}\left(\boldsymbol{\pi}_{k}(i),\right. \text { PLR, ABL, NR, NC) }
$$

The likelihood of not being able to deliver a packet to the video decoder is explained in 4.5 .

The NC and NR values that, whereas satisfying the FEC bitrate constraint, lead to introducing the minimum expected distortion value are picked. The number of video packets to be protected depends on the chosen FEC technique parameters. The most distorting among the $N_{\text {packets }}$ packets in the GOP considering their intrinsic distortion values are selected.

\subsection{Likelihood Of Not Being Able To Recover A Lost Packet}

For a packet not selected to be protected, $P_{\text {loss }}\left(\pi_{k}(i)=0\right)=1$. On the other hand, for protected packets, $P_{\text {loss }}\left(\pi_{k}(i)=1\right)$ is dependent on the size of the generator matrix (NC and NR), and on the parameters that characterize the behavior of the communication channel (PLR and ABL). It reflects the likelihood of losing at least another RTP packet belonging to the same column as the lost packet, or the FEC packet associated to that column.

For obtaining the exact value of $P_{\text {loss }}\left(\pi_{k}(i)=1\right)$, we need to add the contribution of all the possible realizations within the matrix leading to the definitive loss of the packet. This might be a quite time consuming process and thus distance our algorithm from the imposed real-time constraints. Nevertheless, if $\mathrm{NC}$ is set to a large enough value in comparison with $\mathrm{ABL}$, independent loss events can be assumed. In that case, the calculation of the likelihood of not being able to recover a protected packet can be heavily simplified by resorting to the following approximation:

$$
P_{\mathrm{loss}}\left(\boldsymbol{\pi}_{k}(i)=1\right) \simeq \sum_{i=1}^{\mathrm{NR}}\left(\begin{array}{c}
\mathrm{NR} \\
i
\end{array}\right) \operatorname{PLR}^{i}(1-\mathrm{PLR})^{\mathrm{NR}-i}
$$

This equation encloses all the possible realizations in a column that prevent a lost packet in this column from being recovered.

\section{SIMULATIONS AND RESULTS}

For the experiments we have employed the stereoscopic sequence beergarden encoded with the reference software using the GOP prediction structure illustrated in Fig. 1, with hierarchical B pictures and inter-view prediction. The result is a video movie with the following attributes: resolution 1920x 1080 , average bitrate $\sim 4$ Mbps and framerate $25 \mathrm{fps}$. The 6 -second-long sequence has been looped 50 times with the purpose of achieving statistically significant results.

We have compared our SVAP scheme to the following protection strategies:

-Equal Error Protection scheme (EEP): all packets in a GOP are considered identically important. The matrix parameters are set to the maximum values allowed regarding the imposed FEC code rate, in such a way that all packets are protected.

-Frame-Level Unequal Error Protection scheme (FL-UEP): only inter-frame error propagation is taken into account. All packets within a frame are considered equally relevant. The size of the FEC generator matrix is optimized as described for our algorithm.

The packet transmission channel is simulated through a simplified Gilbert model. The ABL values have been selected considering the typical burst length in ADSL networks, that is, about $8 \mathrm{~ms} \mathrm{[14].}$ Furthermore, the extra bitrate, used for FEC packet streaming, is specified as a percentage of the original video bitrate. The total bitrate is obtained as the sum of both.

We present the results in terms of the average peak signal-tonoise ratio (PSNR) in Fig. 4. It can be observed that both unequal error protection schemes clearly outperform the EEP scheme. That means that the designed optimization process that choices the matrix size in function of the system characteristics, and the selection of the most suitable subset of packets to be protected, considering a distortion model, increases the efficiency of the protection scheme, even if overall packet recovery rate decreases. In turn, the proposed strategy obtains better PSNR results than the FL-UEP one, which proves that incorporating information not only about the frame dependencies, but also about the position of packets within frames in the distortion model improves the performance of the protection scheme.

\section{CONCLUSION}

In this paper we have presented a smart UEP scheme for the protection of RTP-wrapped MVC-encoded stereoscopic video streaming in packet networks. The proposed scheme considers the importance of the packet, the behavior of the channel, and the bitrate devoted to protection purposes to both select in real time the most suitable packets to be protected through 1-D interleaved parity AL-FEC and optimize the size of the protection generator matrix. The presented strategy makes use of a packet-level distortion model that determines the relevance of every packet in the video sequence considering different error propagation effects within GOPs.

We have carried out a series of experiments to compare the SVAP scheme with a non-smart EEP strategy and an UEP scheme that just partly consider the proposed distortion model. Simulation results show how the proposed scheme outperforms both strategies thanks to a better management of the use of the limited protection bitrate

\section{ACKNOWLEDGMENT}

This work has been partially supported by the Ministerio de Economía y Competitividad of the Spanish Government under project TEC2010-20412 (Enhanced 3DTV). 


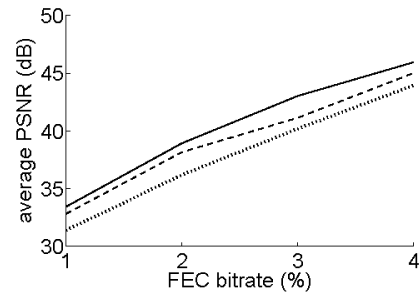

(a) $\mathrm{PER}=0.5 \%, \mathrm{ABL}=4$

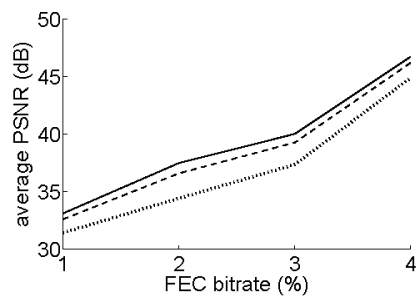

(d) $\mathrm{PER}=0.5 \%, \mathrm{ABL}=8$

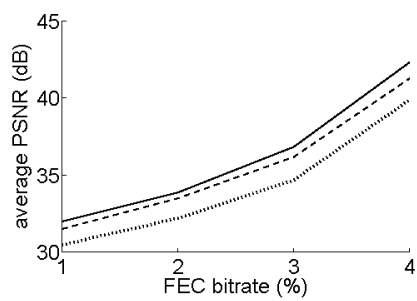

(g) $\mathrm{PER}=0.5 \%, \mathrm{ABL}=12$

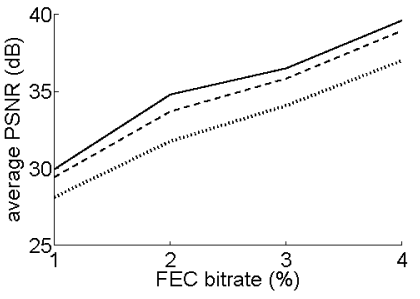

(b) $\mathrm{PER}=1 \%, \mathrm{ABL}=4$

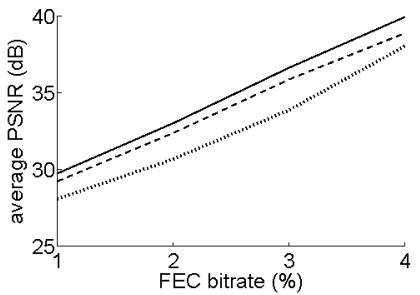

(e) $\mathrm{PER}=1 \%, \mathrm{ABL}=8$

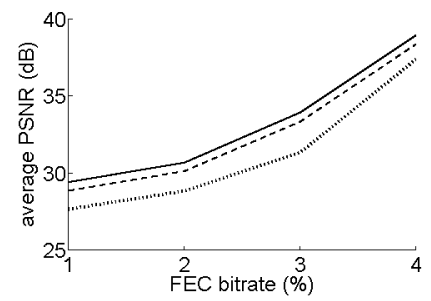

(h) $\mathrm{PER}=1 \%, \mathrm{ABL}=12$

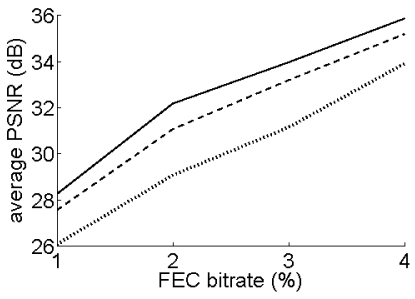

(c) $\mathrm{PER}=1.5 \%, \mathrm{ABL}=4$

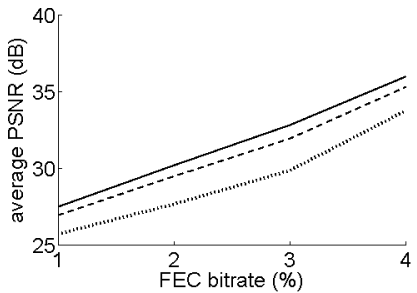

(f) $\mathrm{PER}=1.5 \%, \mathrm{ABL}=8$

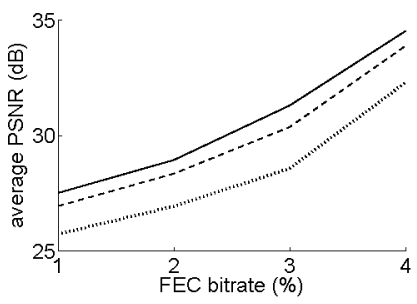

(i) $\mathrm{PER}=1.5 \%, \mathrm{ABL}=12$

Fig. 4. Simulation results: solid lines represent the performance of the SVAP scheme, dashed lines FL-UEP's, and dotted lines EEP's

\section{REFERENCES}

[1] A. Smolic, K. Mueller, N. Stefanoski, J. Ostermann, A. Gotchev, G.B. Akar, G. Triantafyllidis, and A. Koz, "Coding algorithms for 3DTV - A survey," IEEE Trans. on Circuits and Systems for Video Technology, vol. 17, no. 11, pp. 1606 -1621 , Nov. 2007.

[2] A. Begen, T. Akgul, and M. Baugher, "Watching video over the web: part 1: streaming protocols," IEEE Internet Computing, vol. 15, no. 2, pp. 54-63, Mar.-Apr. 2011.

[3] M. M. Hannuksela, V. K. M. Vadakital, and S. JumiskoPyykk, "Comparison of error protection methods for audiovideo broadcast over DVB-H," EURASIP J. Adv. Sig. Proc., 2007.

[4] S. Argyropoulos, A. S. Tan, N. Thomos, E. Arikan, and M.G. Strintzis, "Robust transmission of multi-view video streams using flexible macroblock ordering and systematic lt codes," in 3DTV Conference (3DTV-CON), May 2007, pp. $1-4$.

[5] A. Aksay, D. Bugdayci, and G.B. Akar, "Optimization of encoding and error protection parameters for 3D Video Broadcast over DVB-H," in 3DTV Conference (3DTV-CON), May 2011, pp. $1-4$.

[6] A. S. Tan, A. Aksay, G. B. Akar, and E. Arikan, "Ratedistortion optimization for stereoscopic video streaming with unequal error protection," EURASIP J. Appl. Signal Process., pp. 7:1-7:14, Jan. 2009.

[7] X. Liu, Z. Li, L. Wang, and Z. Chen, "Optimized FEC based on distortion model for MVC streaming," in International Con- ference on Computer Application and System Modeling (ICCASM), Oct. 2010, vol. 4, pp. V4-471 -V4-475.

[8] P. Merkle, A. Smolic, K. Muller, and T. Wiegand, "Efficient prediction structures for multiview video coding," IEEE Trans. on Circuits and Systems for Video Technology, vol. 17, no. 11, pp. $1461-1473$, Nov. 2007.

[9] Pro-MPEG Forum, "Transmission of professional MPEG-2 transport streams over IP networks," Code of Practice 3 release 2, Jul. 2004.

[10] E. N. Gilbert, "Capacity of a burst-noise channel," Bell System Technical Journal, vol. 39, pp. 1253 -1265, Mar. 1960.

[11] J. Gutierrez, P. Perez, F. Jaureguizar, J. Cabrera, and N. Garcia, "Subjective assessment of the impact of transmission errors in 3DTV compared to HDTV," in 3DTV Conference (3DTVCON), May 2011, pp. $1-4$.

[12] M. Barkowsky, K. Wang, R. Cousseau, K. Brunnstroandm, $\mathrm{R}$. Olsson, and P. Le Callet, "Subjective quality assessment of error concealment strategies for 3DTV in the presence of asymmetric transmission errors," in 18th International Packet Video Workshop (PV), Dec. 2010, pp. $193-200$.

[13] DVB, "Application layer FEC evaluations," Document A115, May 2007.

[14] A.C. Begen, "Error control for IPTV over xDSL networks," in 5th IEEE Consumer Communications and Networking Conference (CCNC), Jan. 2008, pp. $632-637$. 